\title{
Giovanni Rotiroti
}

\section{La monstruosité de I'Autre :}

\section{lonesco et la « jeune génération »}

THE MONSTROSITY OF THE OTHER:

IONESCO AND THE "YoUnG GENERATION"

Abstract: This paper is about lonesco's thought on monstrosity, which he associates with politics. Monsters appear in his works when power emerges. In Eugène Ionesco's play, Rhinocéros, what is shown is the transformation of humans into rhinoceroses, but only when they want to join the herd. They are motivated by the desire of freedom, power, and change. This play emphasizes the purposeless and absurdity of the human existence with a critique of the rise of fascism before the Second World War in Europe.

Keywords: Monstrosity; Romanian Literature; Avant-garde Literature; Revolution; Psychoanalysis; Post-Structuralism; Political Emergence; Totalitarianism; Criterion.

\section{GIOVANNI ROTIROTI}

Università degli Studi di Napoli L'Orientale, Italia

rotirotigr@inwind.it

DOI: 10.24193/cechinox.2019.37.18
Hugène Ionesco ne fut pas seulement un
grand homme de théâtre, il fut aussi une grande figure de la littérature universelle. Après la Seconde Guerre mondiale, il a créé une certaine représentation du théâtre par son œuvre imposante, mais aussi une mise en scène de soi et de sa pensée, qui n'est pas sans rappeler ce que Samuel Beckett, Arthur Adamov ou Jean Genet, purent apporter à la figure de "l'écrivain » qui ne cède pas, en termes lacaniens, sur son désir. Mon propos n'est pas d'examiner la singularité de Ionesco, ni de comprendre comment un homme de théâtre devient célèbre. Il s'agit plutôt d'observer la façon dont Ionesco a interrogé sa propre image, les traces qu'il a laissées, les dialogues qui l'ont construit et la façon dont cette expérience réflexive (à tous les sens du terme), éclaire la notion plus générale d'identité post-totalitaire après la chute de l'humanisme.

$\mathrm{Au}$ cœur de sa démarche singulière, la notion d'altérité apparaît comme un pivot dialectique central, la question sur laquelle je veux réfléchir sera le caractère stratégique du monstre et du monstrueux dans la conception politique et théâtrale de Ionesco. Sans s'aventurer dans une synthèse sur l'Autre monstrueux dans la pensée 
ionescienne, on peut tenter de considérer certaines formes d'altérité comme constitutives de son autoportrait littéraire : l'image de soi en tant qu'autre dans le miroir, mais aussi l'autre qui demeure en soi, produit paradoxal d'une déchirure de son for intérieur entre la France et la Roumanie. Le monstre est un pli de la pensée et de l'écriture de Ionesco et indique une pratique de l'esprit et de l'écriture qui n'est jamais la même, qui décentre continuellement son lieu d'énonciation, qui déstabilise les partages et les distinctions quand on essaie de l'identifier.

Pour saisir la complexité du problème de l'altérité chez Ionesco, je me livrerai à un témoignage personnel. Quelques mois après la parution de Non chez Gallimard, j'ai rencontré Eugen Ionesco le 13 novembre 1986 en Italie. À cette date, le célèbre Eugène Ionesco, initiateur du Théâtre de l'Absurde, avait donné une conférence sur le/les Rbinocéros, dans le théâtre de la Badia Fiesolana, à l'Institut Universitaire Européen de San Domenico, non loin de Florence. Je me souviens que l'auteur ne parla pas immédiatement de sa pièce et des motivations profondes qui l'avaient poussé à composer cet ouvrage.

Dans la stupéfaction générale de ceux qui étaient présents dans la salle, sans sourciller et sans donner d'autres indications, Ionesco ouvra son livre $L a$ photo du colonel et commença à lire tout le récit Rhinocéros, pendant plus d'une heure, dans un français impeccable, le français de l'Académie Française. A cette époque, le public présent à cet événement ne savait pas que l'auteur originaire de Slatina était venu régler ses comptes avec la "jeune génération ", c'est-à-dire avec cette génération d'écrivains, penseurs et artistes, notamment la "génération de Criterion ", qui avait été protagoniste avec lui de la vie culturelle roumaine entre les deux guerres. A propos de la "jeune génération " Ionesco écrira ceci en 1987 après son séjour florentin :

On ne compte que quelques survivants de cet immense naufrage. Rari nantes in gurgite vasto. Guerres, maladies, suicides, assassinats, prisons, vieillesse. Qu'est devenue cette jeunesse ? Ces écrivains et ces poètes, et ces génies, cette jeunesse, la " jeune génération », comme elle s'intitulait fièrement, fière d'être jeune, ne s'imaginant pas que la vieillesse, la mort existaient, quelles les attendaient au bout de la route. Où sont les éternellement jeunes, comme ils se croyaient ? Ou, en tout cas, créateurs de chefs-d'œeuvre immortels, " chefs-d'œuvre » oubliés, ensevelis, disparus parmi dizaines de milliers d'autres chefs-d'œuvre, des tas et des tas et des tas de tableaux, de papier, de papiers, des paroles que le vent a emportées, le vent, les tempêtes de l'Histoire ou seulement le temps, ce gouffre implacable, la durée qui use, détruit, déchire, dissout tout. Banalités. Oui, banalités, vérités. Vérités que chacun, chaque génération découvre, progressivement, avec le même étonnement, le même désespoir, la même détresse depuis des siècles, des siècles, des siècles. Et cela aussi, cette découverte est banalité. Vérité, étonnante banalité, inattendue vérité. Dupes que nous sommes ${ }^{1}$.

Quel rapport y a-t-il entre la « jeune génération » et le/les Rbinocéros? Mais, avant tout, que signifie Rhinocéros ? Rhinocéros 
fonctionne comme un nom propre. Cela signifie à la fois rhinocéros au pluriel et rhinocéros au singulier. La langue française maintient cette ambiguïté. Avec cette pièce de 1960, Ionesco revisite un épisode douloureux de sa vie, d'il y a vingt ans. Rhinocéros est donc une réélaboration a posteriori, aprèscoup, quelque chose qui n'arrive qu'après, en retard. Rhinocéros est donc un ouvrage autobiographique sur les années qu'Eugen Ionesco passa en Roumanie à une époque où le nationalisme autochtone se transformait en une véritable hystérie collective, une maladie psychique étrange et inquiétante, une épidémie rhinocérique à grande échelle.

Du point de vue historique, l'idéologie extrémiste de la Légion de l'Archange Michel (qui était un mouvement mystique et profasciste en Roumanie ${ }^{2}$ ) avait commencé à convertir à sa croyance de nombreux intellectuels de la « jeune génération » qui étaient des amis fraternels d'Eugen Ionesco. L'auteur relate cette expérience aliénante dans ses journaux intimes, ses articles, écrits en français et en roumain ${ }^{3}$. Il se souvient que devant ses yeux, les amis ont commencé à montrer des caractéristiques zoomorphes indubitables. C'était une expérience hallucinatoire, la transcription directe d'un délire. Ionesco dit qu'il se sentait de plus en plus seul et isolé des autres, il dit qu'il était entouré par des gens durs comme les pierres, dangereux comme les serpents, implacables comme les tigres. Il se demande : comment peut-on encore parler avec un tigre, avec un cobra? Comment convaincre un loup ou un rhinocéros? Comment être compris par lui ? Il se sentait comme le dernier homme sur une île monstrueuse. A partir de ce moment, il a estimé qu'il ne représentait plus rien pour ses amis, en fait, il était une anomalie pour eux, il était le monstre : « J'avais une conscience de plus en plus mauvaise, malheureuse. Je me sentais un monstre $»^{4}$.

Toujours à travers les journaux intimes de Eugen Ionesco, nous apprenons qu'après que le chef charismatique de la Légion, Corneliu Zelea Codreanu, avait été effectivement tué par ordre du roi - en Roumanie tout le monde avait compris qu'il s'était agi d'un crime d'État - l'auteur observe la propagation d'un virus contagieux, une véritable pandémie psychique avec des contenus idéologiques et de propagande. D'après ce que rapporte Ionesco, cette maladie de l'esprit, en plus quêtre extrêmement contagieuse, n'est pas du tout absurde, mais suit une logique interne précise et spectrale. Tout se passe comme si l'homme, qui a contracté la " rhinocérite ", était possédé par un " cadavre vivant », par un revenant qui agrippe la psyché des intellectuels de la « jeune génération ». Selon Ionesco, ce sont précisément les intellectuels rhinocérisés qui ont historiquement eu la responsabilité de la propagation de la maladie psychique dans la société civile roumaine 5 . Cette culpabilité retombe essentiellement sur ses amis qui ont été enchantés par l'extrémisme politique de la Garde de fer. Voici le long fragment d'une lettre, assez connue par les ionescologues pour son importance historique (mais peu citée pour ses implications politiques), qu'Eugen Ionescu envoya à Tudor Vianu en 1945 :

La génération Criterion, la fière " jeune génération » d’il y a quinze, dix ans, s'est décomposée, a disparu. Aucun d'entre nous n'a pas encore quarante ans - et nous sommes terminés. D'autres, tant, morts.

Votre génération est beaucoup plus chanceuse. Beaucoup plus solide, 
d'ailleurs. Nous avons été des écervelés, des pitoyables. En ce qui me concerne, je ne peux pas me reprocher d'être fasciste. Mais on peut reprocher cela à presque tous les autres : Mihail Sebastian avait gardé un esprit lucide et un honneur authentique. Quel dommage qu'il ne soit plus ! Cioran est ici, exilé. Il admet s'être trompé, dans sa jeunesse. Il m'est difficile de le lui pardonner. Est arrivé ou arrive ces jours Mircea Eliade : pour lui, tout est perdu, du moment où « le communisme a vaincu ". Celui-là est un grand coupable. Mais lui aussi, Cioran aussi, et l'imbécile de Noica, et le gros Vulcănescu, et tant d'autres, (Haig Acterian !, Mihail Polihroniade) sont les victimes de lodieux Nae Ionescu. Si Nae Ionescu nétait pas (ou s'il ne sétait pas embrouillé avec le Roi) nous aurions eu, aujourd'hui, une génération de dirigeants de grande valeur, entre 35 et 40 ans. A cause de lui, ils sont devenus des fascistes. Il a créé une stupide, affreuse Roumanie réactionnaire. Le deuxième coupable est Eliade : à un moment donné il a failli adopter une position de gauche.

Quinze ans sont passés depuis : Haig Acterian, Polihroniade avaient été des communistes. Ils sont morts à cause de leur entêtement et de leur bêtise. Eliade a entraîné lui aussi une partie de " ses collègues de génération » et toute la jeunesse intellectuelle. Nae Ionescu, Mircea Eliade ont été ignoblement écoutés. Qu'en serait-il si ces gens étaient de bons maîtres. A côté d'eux, [Nichifor] Crainic ne compte pas.

À cause de Nae Ionescu, Haig Acterian et Polihroniade sont morts. Et l'imbécile grossier Costin Deleanu et le poète Horia Stamatu sont des fugitifs en Europe (nous les verrons en France un jour), tout comme Eliade, tout comme Cioran, ou Amzăr.

Et les autres imbéciles sont inutilisables : le vaurien Paul Sterian (est-il toujours en Turquie ?), l'enflé Vulcănescu, l'imbécile sec Ion Cantacuzino, l'arrogant, le nigaud, le grandiloquent Dan Botta, l'affecté, l'hypocrite Constantin Noica, l'homme de rien Petru Manoliu. Certains morts à cause de leur bêtise ; d'autres des fugitifs à travers l'Europe, d'autres, heureusement, réduits au silence - toute la génération de Criterion est anéantie. La fatalité les suit tous - également ceux qui ne se sont pas laissés attraper par la stupidité et la folie et ceux restés lucides. Des accidents obscurs et mystérieux ont survenu et les ont jetés eux aussi, au-delà : une difficulté pour Alexandru Vianu, un chauffeur ivrogne pour Sebastian. Ils s'intègrent eux aussi dans le destin commun, ils sont solidaires tacitement. Seul est resté Petru Comarnescu, mais il nétait que l'imprésario, l'organisateur du Criterion, "l'animateur »; il n'en reste plus rien à animer et à organiser. En l'épargnant, le destin a voulu commettre une ironie, mettre davantage en évidence le vide autour de lui.

Quant à moi, j’ai abandonné la partie et j'ai fichu le camp : même si je retournais, puis-je me ré-enraciner ? Je les ai haïs tout le temps, j'ai lutté contre eux, ils mont hai, eux aussi mais sans eux, mes ennemis, je me sens seul. J'étais maudit à les haïr et à 
être attaché à eux : avec qui continuer le dialogue ? Je porte le même signe, moi aussi.

Mihail Sebastian avait été, jadis, mon ennemi (quand il faisait partie de la bande de Mircea Eliade). Il était devenu maintenant un ami, un frère. Qu'aurait-il été utile maintenant : et à la culture roumaine, et à nous, ses amis. Il était devenu mature, grave, profond. Il s'était libéré des doctrines de Mircea Eliade et de Nae Ionescu. Même ainsi je me réjouissais de le voir.

Emil Gulian est-il rentré de Russie ? Est-il libéré ? Ne sont restés même pas quelques rari nantes in gurgite vasto. Il ne reste plus que Comarnescu. [...]

Je me rends compte que c'est bien du point de vue " historique »-qu'il en était ainsi. Le mal était incurable. Il fallait être nettoyé. La maladie nazie, les traditionalismes, "le spécifique ethnique "», la haine contre l'universel - devaient être supprimés aux racines ${ }^{6}$.

Dans ses ouvrages autobiographiques publiés en France, en se référant aux années angoissantes passées dans son pays d'origine, Ionesco notait qu'en Roumanie il y avait une société philosophique extrêmement dangereuse pour la vie démocratique du pays. Cette société de pensée, dirigée par un professeur d'université (Le Logicien du Rbinocéros), s'était convertie à la Légion mystique et appartenait à la famille spirituelle de la Garde de fer. D'après les témoignages de ses étudiants de Bucarest, on sait que ce professeur avait un charisme socratique Après son séjour en Allemagne nazie en 1933, Nae Ionescu laissait présager à ses élus une « communauté politique et spirituelle » de droite qui aurait dû capter le malaise social provenant des masses de jeunes cultivés sans emploi et mécontents de la vie civile qu'ils menaient dans leur pays ${ }^{7}$. Cette « communauté spirituelle et politique » aurait donc dû transformer ce malaise juvénile en sens révolutionnaire, pour la rédemption de la patrie. En fait, cela aurait été une révolution anti-institutionnelle et anti-libérale. Ionesco dit que les représentants de cette communauté philosophique et politique ont envahi tous les journaux, les revues et la radio. Ils donnent des cours à l'université, organisent des conférences dans les théâtres de la capitale et de la province, écrivent des livres, parlent, parlent et couvrent tout avec leur vacarme. Leur vision du monde est devenue dominante et ceux qui ne se soumettent pas à eux seront perdus.

Il existe en Roumanie une société philosophique portant le nom d'un professeur fasciste et qui groupe soixante jeunes philosophes. Nous savons combien sont dangereuses, efficaces, ces sociétés de pensée. Ces soixante ou soixante-dix idéologues se réunissent, discutent, se préparent : ils sont « mystiques ", légionnaires ou pré-légionnaires, c'est-à-dire membres de la famille spirituelle Garde de fer, des germes, des ferments, ils sont soixantedix, ils deviendront cent, deux cents mille, ils envahissent les journaux, les revues. Ils donnent des cours à la Faculté, des conférences, écrivent des livres, parlent, leurs voix couvrent tout. Ce sont les conséquences de l'Histoire. Leur vision du monde est en train de vaincre. Alors, nous serons perdus ${ }^{8}$.

La situation dégénère en Roumanie et donne lieu à de terribles excès. Le roi 
impose en fait une dictature personnelle. Il adopte des mesures antisémites et rend la peine de mort exécutoire. Si d'une part le souverain avait combattu la secte mystique de la Légion, d'autre part il utilise à ses propres fins la propagande idéologique qui avait été faite au nom du génie éternel de Codreanu. Le fantasme du capitaine survit ainsi à ses restes mortels. Alors que le corps physique de Codreanu est un cadavre, l'autre, le « spirituel », survit dans sa dimension spectrale et fantasmatique. Eugen Ionescu est angoissé. Il se sent seul et abandonné par ses vieux amis de Criterion. Il essaye d'échapper à la Roumanie. Il dit que tout pouvait lui arriver : une possible contamination idéologique, une métamorphose définitive comme ses amis, devenir un chien, être aussi habité par le " cadavre vivant » du Capitaine de la Légion.

Bérenger dans la pièce vacille, le conformisme, pense-t-il, pourrait être plus sûr. Il ne peut pas faire comme les autres. Et il ne sait même pas pourquoi. C'est la panique la plus totale. L'héroïsme de Bérenger, dans la pièce et dans le récit, est montré comme involontaire. Le choix est donc inconscient. C'est le trait éthique ionescien du Rhinocéros. Ce n'est donc pas un calcul ou une décision consciente. Mortifié par sa propre faiblesse, Eugen Ionesco, à travers Bérenger, refuse tout simplement dêtre autre chose que ce quil est. Mais il ne connaît pas la cause ou la raison. Quelque chose, apparemment singulière ou symptomatique, l'empêche de rejoindre les coryphées de la Légion. Peut-être qu'il n'a pas leurs qualités, il n'est pas aussi bon qu'eux :

Je découvris que leurs barrissements avaient tout de même un certain charme, un peu âpre certes. J'avais dû m'en apercevoir quand il était temps. J'essayai de barrir : que c'était faible, comme cela manquait de vigueur ! Quand je faisais un effort plus grand, je ne parvenais qu'à hurler. Les hurlements ne sont pas des barrissements ${ }^{9}$.

Ou ce sont plutôt les barrissements, c'est-à-dire les slogans, les mots d'ordre, les prescriptions, le passage à l'acte politique des rhinocéros qui l'empêchent de s'adapter à la « communauté politique » de ses amis. Peut-être est-ce précisément certains mots, un certain langage, un certain son pervers qui font que le protagoniste se retire de la vision dominante, apparemment conciliante, normalisatrice et conformiste. Ils sont tous d'accord pour faire la révolution nationale. Tout le monde dit la même chose. C'est un refrain qui le hante continuellement.

- Voici un slogan rhinocérique, écrit-il, un slogan « d'homme nouveau ", qu'un homme ne peut comprendre : tout pour l'État, tout pour la nation, tout pour la Race. Cela me paraît monstrueux, évidemment. [...] Mais qu'est-ce que l'État, comment peut-on se projeter dans l'État, et quest-ce que la Nation et qu'est-ce que la Société ? Des abstractions, dépersonnalisantes, non existentielles, puantes, suprêmement aliénantes. L'humanité n'existe pas. Il y a des hommes. La société n'existe pas, il y a des amis. Pour un rhinocéros, ce n'est pas la même chose. Pour moi, son État est un fantasme. Pour lui, c'est la personne concrète qui est un fantasme ${ }^{10}$.

Encore le fantasme de la Légion, la logique spectrale du Rbinocéros, la mentalité 
fasciste des Gardes de fer et de leur collectivisme. Les paroles de Ionesco reflètent l'affection animale qui naît de la force souveraine de la loi, c'est-à-dire de la peur, de la terreur généralisée. Le monstre est l'Autre du sujet, ce que le sujet ne doit pas être pour devenir un véritable sujet. Le monstre est l'autre de la vérité, il nest pas simplement une erreur. Un mot rhinocérique apparaît lorsque la loi morale devient un dispositif pervers. Cette loi exige une forme absolue d'obéissance en échange d'une protection. Nous assistons à l'exaspération du monstre, de l'animal, de la bête et de la souveraineté du pouvoir. Les figures de la loi, de l'État-Nation, de la panique souveraine sont des émanations bestiales. Le " démon des légionnaires " qui donne la voix à l'appel des Gardes de fer, est en réalité l'incarnation d'un mort. La mort incarne en effet l'altérité absolue pour Ionesco. Cette approche de la mort et du mort indique que nous sommes en relation avec quelque chose qui est absolument autre. L'artifice pervers de la loi spectrale incarné par un mort transforme la vie sociale en machineries sadiques sur un fond mythique et xénophobe. C'est une logique implacable et obscène, un mécanisme non régulé. C'est un besoin d'absolu qui se nourrit d'un salut surnaturel. L'offrande à ce «Dieu obscur » revendique le sacrifice suprême, qui nest pas seulement celui de la vie réelle, de l'organisme biologique, mais c'est avant tout la mort de la parole, la fin de l'être humain. Les rhinocéros ne parlent pas, ils crient, ils sont capturés par un dispositif pervers qui les pousse à une convulsion à la fois motrice et mortelle. La loi et le crime dans cette logique sont indissolubles et soumis à un pouvoir absolu, qui ne permet pas de répliques. Nous lisons dans le journal de Ionesco :
Regardez-les ; écoutez-les : ils ne se vengent pas, ils punissent. Ils ne tuent pas, ils se défendent : la défense est légitime. Ils ne haïssent pas, ils ne persécutent pas, ils rendent justice. Ils ne veulent pas conquérir ni dominer, ils veulent organiser le monde. Ils ne veulent pas chasser les tyrans pour prendre leur place, ils veulent établir lordre vrai. Ils ne font que de saintes guerres. Ils ont les mains pleines de sang, ils sont hideux, ils sont féroces, ils ont des têtes d'animaux, ils s'enfoncent dans la boue, ils hurlent. Je ne veux pas vivre avec ces fous, je ne suis pas de leurs fêtes, ils veulent m'entraîner de force avec eux. Pas le temps de leur expliquer ${ }^{12}$.

Ce que Ionesco met en scène dans son œuvre n'est pas seulement la dénonciation de la contagion idéologique, mais c'est avant tout la pulsion du sujet acéphale qui pousse les coryphées de la Légion vers la compétition spasmodique, le fanatisme, le cynisme, la voracité du pouvoir masqué par l'idéalisme religieux et configuré comme un savoir infaillible. Tout cela avant que la véritable dictature s'installe dans les ganglions atrophiés de la pensée et assume la caractéristique définitive de la mutation psycho-anthropologique.

Ionesco établit ainsi une équivalence entre l'« homme nouveau » du totalitarisme idéologique et Rhinocéros, c'est-à-dire l'invention du fantasme subjectif déterminé par la «panique politique ». Pour Ionesco, l'homme nouveau, mis en avant par l'idéologie totalitaire, est un mort-vivant, le « cadavre vivant » dans sa dimension spectrale. C'est l'identification au héros parfait de la Garde de fer qui sacrifie sa vie pour la 
Nation. Le Réel poursuivi par l'homme nouveau est horrible et inoui, mystique et instinctif. C'est un nouveau type de sujet enterré vivant, qui abolit l'ancien régime après la chute de l'humanisme et de la personne morale. En suivant cette logique du sacrifice jusqu'au bout, la mutation anthropologique d'un tel sujet ne peut avoir lieu qu'à la condition de la destruction de soi et de l'autre. Le sujet rhinocérisé qui a été contaminé par la rbinocérite se retrouve donc dans un no man's land, dans le royaume de la mort réelle où tout ordre symbolique est suspendu et où toutes les différences sont abolies au nom d'une logique implacable qui élimine tout ce qui apparaît comme un corps étranger dans l'imaginaire social de la Nation. Les amis d'Eugen Ionesco, sous ses yeux, sont devenus méconnaissables :

J'ai assisté à des mutations. J'ai vu des gens se transformer, à peu près sous mes yeux. C'est comme si j'avais surpris le processus même de la métamorphose, comme si j’y avais assisté. Je les sentais d'abord devenir de plus en plus étrangers, j'ai senti comment germait en eux une autre âme, un autre esprit. Ils perdaient leur personnalité, remplacée par un autre. Ils devenaient autres $^{13}$.

Ces amis sont devenus des fantasmes, des fantômes d'animaux ou des animaux-fantômes. Mais le « souvenir lointain ", l'« énorme déception » qui semble l'immerger «dans les ténèbres sans fin » est celle liée à « A. », c'est-à-dire à l'Autre, à son collègue de la « jeune génération " Arşavir Acterian sans doute. Voici le témoignage de la désorientation ionescienne à la vue de l'ami fraternel, devenu inexorablement autre, pris dans l'engrenage monstrueux du dispositif infernal de la Garde de fer.

J'ai rencontré dernièrement $\mathrm{A}$. On ne peut plus s'entendre, c'est un autre, un autre qui porte le même nom. Autrefois, il n'y a pas longtemps, quand je prononçais son nom, quand jeécrivais son nom, quand je le voyais, ou quand je pensais à lui, une lumière se répandait dans mon cœur; je sentais une présence chaleureuse, réconfortante. Je ne me sentais plus seul. Maintenant, lorsque j'écris son nom, lorsque je prononce son nom, lorsque je revois, en esprit, son visage, c'est l'horreur, presque la haine, un grand malaise. Son nom semblait être un nom d'archange. Maintenant, ce même nom est barbare, ou il me semble l'être : pire, c'est le nom d'une hyène ou d'un chien.

Il y a quelque temps, il habitait une petite maison au milieu d'un immense jardin. Le jardin était gardé par un énorme chien, un bouledogue, presque sauvage, très laid, d'une cruauté stupide. Il était enchaîné dans la journée, il ne s'entendait avec personne. Implacable et féroce. C'est le seul chien vraiment idiot que j'aie jamais connu. Il n'aboyait jamais. Il sautait silencieusement sur quiconque pour le mettre en lambeaux. Une fois, dans la journée, le chien avait réussi à défaire sa chaîne. A. se trouva face à face avec le chien. Le chien sauta sur lui. A., criant, a lutté quelques minutes avec le chien, dans un combat atroce. Quand on a réussi à les séparer, A. était blême, le visage d'un autre, changé, il était curieusement changé. C'est à partir 
de ce moment-là que A. a commencé dêtre un autre. Progressivement, quelqu'un d'autre l'a remplacé. La bête l'avait possédé, elle avait laissé en lui sa semence. J'ai rencontré, par hasard, récemment, le nouveau $A$. J'étais stupéfié, effrayé. La germination, la croissance, l'épanouissement du germe de la bête est accompli. La figure même de A. n'est plus la même : son visage s'est élargi, il ressemble au chien. Il est devenu l'enfant du chien, ou peutêtre la femelle de la bête. Il est féroce, implacable, stupide. On ne peut plus lui parler. Il ne comprend plus ma langue, son ancienne langue. Je lui ai dit, brutalement, un seul mot : «quon devrait l'exterminer ». En effet, il n'a plus qu'à peine l'apparence de A. - A. nest plus qu'un souvenir lointain ${ }^{14}$.

Ainsi, en ce qui concerne la question complexe de la monstruosité de l'Autre, tous les amis nont pas pu résister à la contagion psychique rapportée par Ionesco. Que se passe-t-il lorsque vous tombez dans la réalité et que cette réalité s'appelle Hitler, Mussolini ou Codreanu ? Nous sommes ouverts au gaspillage, à la dépense, à la dissipation de mots marqués par leur pouvoir de destruction, et nous assistons au spectacle d'une transformation non seulement linguistique, mais surtout anthropologique.

Dans ce cas, la parole par rapport à Autrui n'est plus une question d'amour ou d'amitié. Ce n'est plus le désir ou le vide qui parle et demande, mais la violence insensée et aveugle, la terreur à l'état pur. Le délire de la rhinocérite parle avec un certain langage, témoigne de la perte de l'homme où tout est mensonge. Mais nous prétendons vivre dans la vérité absolue et irréfutable des faits. Voici le pouvoir destructeur du mot. Ce monde est devenu intolérable parce que les choses ne semblent pas du tout satisfaisantes. Donc on a besoin de la réponse paranoïaque qui donne la certitude. On a besoin de l'absolu, du délire, qui garantit d'une certaine manière un plan sûr de salvation. C'est la vraie folie de la rhinocérite. Il suffirait donc de rester logique jusqu'au bout pour y trouver une destination finale, une quiétude apaisante, une sérénité épanouie à laquelle on aspire.

Philippe Lacoue-Labarthe et JeanLuc Nancy, qui ont étudié attentivement la Psychologie des masses et analyse du moi et L'homme Moïse et la religion monothéiste de Sigmund Freud, ont tenté de déchiffrer la "vérité » de la "panique politique ", dont a souffert sans aucun doute Ionesco en Roumanie, en identifiant une zone de frontière entre psychanalyse et retrait du politique. Les deux auteurs en dialogue ont trouvé le signe d'une problématique dans le processus de « dépropriation du sujet de la culture, de la société et de la politique » par rapport à Autrui :

Autrui n'« est » jamais tout d'abord que son propre retrait, que son propre-impropre retrait, le retrait de son amour - un amour qui nest peut-être luimême que la forme ou le creusement de ce retrait (non pas amour d'objet, ni de sujet mais ce retrait à partir de quoi il peut y avoir objet : à l'origine, ce n'est pas tant d'une perte d'objet qu'il faut parler, ni d'ailleurs d'une perte d'amour, mais de ce retrait qui fait l'amour). Autrui n'est pas d'abord l'autre identique, mais le retrait de cette identité - l'altération d'origine. Narcisse, pour autant qu'il puisse être, 
n'est jamais qu'entamé par ce retrait (ce qui sans doute engage tout autre chose que le concept simplement négatif et dramatique de "blessure narcissique $»$ ).

[...] Si la naissance de la société n'est autre que celle de l'individu, et réciproquement (ainsi que Rousseau, Hegel et Marx l'ont déjà su avec précision), et si le tabou des morts approche l'explication du totémisme, alors le « meurtre du père " n'est un meurtre que dans la mesure où la mort d'autrui (la mort du mort, le mort comme mort) est ce retrait d'amour : et il ne saurait y avoir de Père qu'après la mort du (premier) mort. De cette manière encore, rien ne commence par l'affrontement $d u$ Narcisse absolu : tout commence, au contraire, dans l'entame infiniment originaire des narcisses, pour quoi se scelle leur non-rapport.

Le Père, c'est le mort re-présenté, ou re-présentifié : c'est la mimèsis cathartique du retrait d'amour (et de la haine), et qui oblige en effet au meurtre sacrificiel indéfiniment répété. L'interdiction de représenter, essentielle au monothéisme d'Ikhnaton/Moïse, c'est donc l'interdiction de tuer, qui revient donc lui-même à l'interdiction de transgresser le retrait d'amour, ou l'amour dans son retrait : qui revient à la nécessité de l'entame (Freud la nomme : Anankê). Le monothéisme n'aborde pas un dieu (une figure de la mort) [...], il affronte la mort, autrui, - mon désir -, visage retiré de toutes les figures ${ }^{15}$.

A l'origine, de ce point de vue, la « panique politique » n'est donc pas une simple maladie psychique mais une question ontologique, c'est-à-dire une " situation à la fois originale et actuelle ${ }^{16}$. La passion archéphilique de Freud rencontre dans l'arkhê le principe de l'histoire, du politique et du commencement, de la remontée vers l'origine, mais aussi le principe du commandement que le même mot désigne, celui de l'ordre ou de l'agencement selon une loi. Ce qui fera dire à Jacques Derrida que «[...] nul mieux que Freud n'a éclairé ce que nous avons appelé le principe archontique de l'archive, ce qui en elle suppose non l'arkhê originaire mais l'arkhê nomologique de la loi, de l'institution, de la domiciliation, de la filiation. Nul mieux que lui n'a analysé, c'est-à-dire a aussi déconstruit l'autorité du principe archontique. Nul mieux que lui n'a montré comment ce principe archontique, c'est-à-dire paternel et patriarchique, ne se posait qu'à se répéter et ne revenait pour se re-poser que dans le parricide. Il revient au parricide refoulé ou réprimé, dans le nom du père comme père mort ${ }^{17}$.

L'absence, c'est-à-dire le parricide refoulé du père, engendre la " panique politique ». Selon Philippe Lacoue-Labarthe et Jean-Luc Nancy :

La panique a lieu dans le retrait de cette naissance. Dissolution du Politique, elle révèle l'absence, et plus que l'absence du Père-Pan. On peut alors reconstituer le Politique, la simulation cathartique et réappropriative de la perte du Narcisse. Ou bien, puisqu'on vient ainsi de définir d'abord la Religion : le Politique est la volonté de l'intégrale appropriation de cette simulation elle-même ; devenir profane de la religion, il présuppose ici même (et non dans le ciel) donné le 
Sujet : l'homme doué de parole et de sens moral que pose Aristote au début de sa Politique. L'animal politique s'y sacrifie lui-même son image ${ }^{18}$.

$\mathrm{Au}$ croisement de l'identification et du narcissisme qui redéfinissent la place de l'Autre dans la formation de la société, c'est-à-dire à partir de l'intersection entre psyché individuelle et vie collective, Nancy et Lacoue-Labarthe ont saisi la relation fragile qui existe entre " retrait du politique " et origine de la " panique politique ». Tout cela nest pas sans rapport avec l'expérience qu'Eugen Ionesco a vécu au sein de la "génération de Criterion ».

Ionesco a identifié le noyau phantasmatique plus persistant de la "panique politique » dans le phénomène du balkanisme. Ce noyau phantasmatique semble être précisément celui ethnique, religieux et politique, historiquement représenté par la Garde de Fer en Roumanie, c'est-à-dire l'Autre monstrueux : «Le phénomène Garde de fer n'est pas quelque chose passager, c'est profondément balkanique, c'est vraiment l'expression de la dureté de l'âme balkanique sans raffinements $»^{19}$. Dans l'après-coup, Rbinocéros n'est pas lisible en dehors de la culture et de l'imaginaire des Balkans. Rhinocéros est le symptôme de la "panique politique » qu'Ionesco a souffert en Roumanie lorsque la « jeune génération " s'est décomposée, a disparu. De ce point de vue, Rbinocéros est un symptôme qui se manifeste comme crise et retour du fantasme même de « l'âme balkanique », qui nous amène à constater que le symptôme n'est plus sur le devant de la scène. En revanche, l'angoisse est d'emblée mise en avant. Reconnaître et supporter cette angoisse primordiale a permis à Ionesco d'élaborer ou de réécrire un symptôme par l'intermédiaire du fantasme. Le scénario de la phobie rhinocérique implique une fixité, un récit, un déroulement réglé par la scène théâtrale ; en même temps, le nom même de Rbinocéros introduit la notion de phobie constitutive à la structure fantasmatique et rend compte soit d'un impossible à dire, soit d'un envahissement par l'angoisse où le sujet s'effraie en quelque sorte de ce qui le submerge. L'angoisse peut constituer une voie d'accès au versant réel du symptôme de la panique. Elle peut permettre de toucher le point de jouissance du symptôme. L'appui que le symptôme prend dans le fantasme peut être mis à jour. Ainsi se construira une formule du désir ou du fantasme, soutien du désir, pouvant arrêter ce cheminement incessant du sujet à la dérive ${ }^{20}$. Le phénomène de la Garde de fer peut être reconduit donc, de ce point de vue, à la puissance du Réel exprimé par le fanatisme religieux, l'antisémitisme, le fondamentalisme xénophobe et ultranationaliste, c'est-à-dire il doit être interprété comme un excès sans loi, au sens lacanien, qui ne peut pas être subjugué, ni réglementé par quelque « Nom du Père » ${ }^{21}$. En retraversant cette expérience d'angoisse à travers le Rbinocéros, Ionesco a réussi à mettre à nu la définition de l'ambivalence de « l'âme balkanique », en particulier en ce qui concerne son glissement dans une forme politique inquiétante et un acte subversif qui a été réalisé par la main armée et meurtrière de la Garde de Fer.

À Florence, au cours de sa conférence sur Rhinocéros en 1986, Ionesco avait dit que quand presque tout le monde était devenu Garde de fer, un jour l'un de ses amis, en lui disant que les légionnaires n'avaient finalement pas tort de critiquer les Juifs d'un certain point de vue, ce jour-là, dit-il, se rendit compte qu'il ne pouvait pas « vivre 
avec ces gens » et décida qu'il aurait fait tout pour échapper à sa condition horrifiante, car il se sentait de plus en plus isolé et incompris. Puis il ajouta : "Je suis allé dans mon pays d'enfance. Là je n'étais pas seul, cela m’a sauvé ».

\section{Notes}

1. Eugène Ionesco, La quête intermittente, Paris, Gallimard, 1987, pp. 10-11.

2. Cf. Traian Sandu, Un fascisme roumain. Histoire de la Garde de fer, Paris, Perrin, 2014.

3. On peut voir à ce propos les articles d'Eugène Ionesco contenus dans les ouvrages: Eu, ediție îngrijită de Mariana Vartic. Cu un prolog la Englezește fără profesor de Gelu Ionescu și un epilog de Ion Vartic, Cluj, Editura Echinox, 1990 ; Război cu toată lumea. Publicistică românească, vol. I-II, ediție îngrijită și bibliografie de Mariana Vartic și Aurel Sasu, București, Editura Humanitas, 1992 ; Scrisori către Tudor Vianu, II, 1936-1949, ediție îngrijită de Maria Alexandrescu Vianu şi Vlad Alexandrescu, note de Vlad alexandrescu, Bucureşti, Editura Minerva, 1994 ; Manuscriptum, ediție îngrijită de Simona Cioculescu, 1-2, 1998, pp. 205-226 ; Texte recuperate, Caiete critice, nn. 1-2-3, 2009 ; Texte recuperate (II), Caiete critice n. 4, 2009 ; Texte recuperate, Caiete critice, nn. 5-6-7, 2009 ; Texte recuperate, Caiete critice, nn. 8-9, 2009 ; Texte recuperate, texte regăsite, Caiete critice, nn. 10-11-12, 2009. En français on peut voir aussi les volumes d'Ionesco : Journal en miettes, Paris, Mercure de France, 1967 ; Présent-Passé, Passé-Présent, Paris, Mercure de France, 1968 ; Antidotes, Paris, Gallimard, 1977 ; Un homme en question, Paris, Gallimard, 1979 ; Ruptures de silence, Paris, Mercure de France, 1995. Ouvrages universitaires et essais consultés : Zigu Ornea, Anii treizeci : extrema dreaptă romanească, Bucureşti, Editura Fundaţiei culturale române, 1995 ; Marta Petreu, Ionesco în ţara tatălui, Cluj-Napoca, Editura Biblioteca Apostrof, 2001 ; Matei Călinescu, Eugène Ionesco. Teme identitare şi existenţiale, Iaşi, Editura Junimea, 2006; Giovanni Rotiroti, Odontotyrannos. Ionesco e il fantasma del Rinoceronte, Rome, Il Filo, 2009 et Il segreto interdetto. Eliade, Cioran e Ionesco sulla scena comunitaria dell'esilio, Pise, Edizioni ETS, 2011.

4. Eugène Ionesco, La photo du colonel, Paris, Gallimard, 1962, p. 128.

5. Cf. Eugène Ionesco, Notes et contre-notes, Paris, Gallimard, 1962.

6. Cf. Bogdan Ghita, Eugène Ionesco un chemin entre deux langues, deux littératures, Paris, LHarmattan, 2011, pp. 271-272.

7. Cf. Giovanni Rotiroti, La Comunità senza destino. Ionesco, Eliade, Cioran all'ombra di Criterion, Florence, Alefbet, 2007.

8. Eugène Ionesco, Présent passé, Passé présent, p. 163.

9. Eugène Ionesco, La photo du colonel, p. 127.

10. Eugène Ionesco, Présent passé, Passé présent, p. 115-116.

11. Eugen Ionescu, Război cu toată lumea, Vol. II, p. 273.

12. Eugène Ionesco, Présent passé, Passé présent, p. 75.

13. Ibid, p. 168.

14. Ibid., p. 172-173.

15. Philippe Lacoue-Labarthe et Jean-Luc Nancy, La panique politique suivi de Le peuple juifne rêve pas, Paris, Christian Bourgois Éditeur, 2013, p. 53-54.

16. Alberto Zino et Costanza Tabacco, Prefazione, in Philippe Lacoue-Labarthe, Jean-Luc Nancy, Il panico politico, Pise, Edizioni ETS, 2018, p. 8.

17. Jacques Derrida, Mal d'Archive. Une impression freudienne, Paris, Galilée, 1995, p. 147-148.

18. Philippe Lacoue-Labarthe et Jean-Luc Nancy, La panique politique suivi de Le peuple juif ne rêve pas, p. 57.

19. Eugène Ionesco, Présent passé, Passé présent, p. 182.

20. Cf. Brigitte Haie, « De l'angoisse au symptôme », Figures de la psychanalyse 2010/1 (n 19), p. 119-129.

21. Cf. Jacques Lacan, Séminaire, III, Les psychoses, 1955-1956, Paris, Seuil, 1981. 